\title{
Possible phase diagrams for reversibly interpenetrating polymer networks
}

\author{
$\operatorname{AUTHOR}(\mathrm{S})$ : \\ Tanaka, Fumihiko
}

\section{CITATION:}

Tanaka, Fumihiko. Possible phase diagrams for reversibly interpenetrating polymer networks. Physical Review Letters 1992, 68(21):3188-3191

\section{ISSUE DATE:}

1992-05-25

URL:

http://hdl.handle.net/2433/49181

RIGHT:

Copyright 1992 American Physical Society 


\title{
Possible Phase Diagrams for Reversibly Interpenetrating Polymer Networks
}

\author{
Fumihiko Tanaka \\ Department of Physics, Faculty of General Education, Tokyo University of Agriculture and Technology. \\ Fuchu-shi, Tokyo 183, Japan \\ (Received 16 September 1991)
}

\begin{abstract}
We present possible equilibrium phase diagrams on the temperature-concentration plane for binary interpenetrating polymer networks (IPN) in which network junctions are formed by reversible crosslinking. It is shown that, in most parts of the phase plane, the formed networks of both species are mutually exclusive so that the system demixes into small domains of heterophases, but that there is a small region just above the top of the miscibility gap in which a molecularly mixed IPN can stay in a stable phase. The condition for the existence of a stable IPN is examined. Competition between network formation and two-phase demixing is studied.
\end{abstract}

PACS numbers: $64.70 .-p, 64.75 .+\mathrm{g}, 82.70 . \mathrm{Gg}$

Interpenetrating polymer networks (IPN's) are defined as a nonbonded but unseparable combination of two polymers, each in network form. Like most other multicomponent polymer materials, IPN's usually phase separate due to their small entropy of mixing, but the presence of cross-links leads to a reduction of the domain size. As a consequence of the complex dynamic balance between the two opposite tendencies, the synthesis of IPN's can produce materials ranging from molecularly homogeneous ones to microscopically phase-separated ones with phase domains of various sizes, hence yielding a unique method of controlling the morphology and mechanical properties. The chemistry and physics of IPN's are extensively reviewed in Refs. [1-3].

In this paper we consider physically cross-linked networks in which junctions are formed by noncovalent associative forces. The junctions are assumed to be sufficiently weak to break and recombine in thermal fluctuations. These attractive forces include [3] hydrogen bonding in aqueous systems, ionic association in ionomeric IPN's, and cross-linking through complex formation in block-type copolymer networks. In such a weakly connected IPN, polymers can easily reach equilibrium by the reorganization (creation and annihilation) of the junctions in the course of the phase separation, and, under certain conditions, the possibility arises that a molecularly homogeneous phase comprised of the two unseparable networks is stabilized. Although no systematic observation of the reversible IPN has yet been reported, the present paper aims at exploring the possible types of phase diagrams, to find the conditions for the appearance of a stable IPN.

The phase stability of weakly cross-linked IPN's was theoretically studied [4] under the assumption of chemical quenching - that is, the kinetics of polymerization and cross-linking is so rapid that any phase separation during these processes is negligible. Although the free energy of the IPN was constructed on the basis of the Flory theory of rubber elasticity [5] in order to study the stability limit (i.e., spinodals), competition between network formation (or gelation) and phase separation was not studied.

In addition to the gelation and the macroscopic phase separation, microphase formation can simultaneously coexist in IPN because the topological constraints in the networks play a similar role to the block copolymer junctions. The effect of the reversible microphase formation on the macroscopic phase separation was recently studied by the present author [6] for the simplest case of the diblock copolymers. The calculation of the scattering function developed in the study is directly applicable to the stability analysis of the heteropolymer networks against fluctuations of finite wavelength, but a detailed analysis is beyond the scope of this paper.

There are two main methods of synthesizing IPN's: simultaneous IPN (sim-IPN) and sequential IPN (seqIPN). For sim-IPN, functional monomers (or primary chains) of both species are mixed together and polymerized. For seq-IPN, a polymer network of species $A$ is synthesized, and functional monomers $B$ are swollen into the network and polymerized. We therefore consider the following model system.

Consider a binary mixture of linear polymers $A$ and $B$. The number of statistical units (whose sizes are assumed to be the same for both species and given by $a$ ) on a chain is $n_{A}$ and $n_{B}$ for each species. In the case of monomer reactions, we must set $n_{A}=n_{B}=1$. Assume that an $A$ chain carries $f$ functional groups capable of forming pairwise reversible bonds, and a $B$-chain, $g$ functional groups. Functional groups on $A$-chains and $B$-chains are assumed to be nonreactive with each other. In thermal equilibrium, we have a distribution of $A$-clusters and $B$-clusters in the system. Let $V$ be the total volume of the system, and let $\Omega \equiv V / a^{3}$ be the number of microscopic cells on which the statistical units are placed within the framework of the Flory-Huggins lattice theory [7] of polymer mixtures. Let $N_{i}^{A}\left(N_{m}^{B}\right)$ be the number of clusters made up of $l A$ chains ( $m B$-chains). The volume fraction occupied by the clusters made up of $I A$-chains ( $m B$-chains) is then given by $\phi_{i}^{A}=\ln _{A} v_{l}^{A}\left(\phi_{m}^{B}=m n_{B} v_{m}^{B}\right)$, where $v_{l}^{A} \equiv N_{i}^{A} / \Omega$ $\left(v_{m}^{B} \equiv N_{m}^{B} / \Omega\right)$ is the number concentration of the clusters. In addition to these finite-size clusters, macroclusters 
(networks, or gels) can appear for $f \geq 3$ (and/or $g \geq 3$ ). Let $\phi$ be the total composition of species $A$. (The composition of species $B$ is given by $1-\phi$.) The volume fraction of $A$-gel $\left(B\right.$-gel) is then given by $\phi_{A}^{G}=\phi-\sum_{l=1}^{\infty} \phi_{l}^{A}$ $\left(\phi_{B}^{G}=1-\phi-\sum_{m=1}^{\infty} \phi_{m}^{B}\right)$. The equilibrium thermodynamics of network-forming polymer mixtures was developed by the present author [8] to study the interrelation between structure formation and phase separation. The present study is based on the general framework formulated in the theory.

The free-energy change $\Delta F$ to bring the system from the starting reference state-that is, the state where all unreacted monomers (or primary chains) are prepared separately - to the actual equilibrium state can be written as

$$
\begin{aligned}
\frac{\beta \Delta F}{\Omega}= & \gamma A(\phi)+\xi\left[\sum_{l=1}^{\infty}\left(\Delta i^{A}+\ln \phi_{i}^{A}\right) v_{i}^{A}+\delta_{A} v_{A}^{G}\right] \\
& +\sum_{m=1}^{\infty}\left(\Delta_{m}^{B}+\ln \phi_{m}^{B}\right) v_{m}^{B}+\delta_{B} v_{B}^{G}+\chi \phi(1-\phi),
\end{aligned}
$$

where $\beta=1 / k T$ is the reciprocal of the temperature, and $\chi$ is the Flory interaction parameter. Here the first term describes the elastic free energy of the network due to swelling. In the case of seq-IPN, an $A$-network, which is permanently cross-linked in a dry state $\left(\phi_{c}=1\right)$, is prepared, and then monomers $B$ are swollen into the network, inside which they form clusters. Hence we have $\gamma>0$, where $\gamma$ is the number of elastically active chains (per unit cell) in the prepared $A$-network. The elastic energy of swelling is given by

$$
A(\phi)=\frac{3}{2}\left(\alpha^{2}-1\right)-3 B \ln \alpha,
$$

where the swelling ratio $\alpha$ is defined by $\alpha \equiv\left(\phi_{c} / \phi\right)^{1 / 3}$. Numerical values of the coefficient $B$ differ depending on the various theoretical descriptions: $B=0$ in the JamesGuth [9] phantom network theory, $B=2 / f$ by Flory and co-workers [10], and $B=1$ by Kuhn and Grün [11]. Since arguments still remain, we leave $B$ as a parameter. In a sim-IPN, both networks are formed in the undeformed state, so that we have no elastic free energy $(\gamma=0)$.

In the second term of Eq. (1), $\Delta_{i}^{A} \equiv \beta\left(\mu_{l, A}^{0}-l \mu_{A}^{0}\right)$ is the free-energy change produced in the process of singlecluster formation-that is, the free energy produced when $l$ unreacted $A$-chains form a single cluster of size $l$. (Each $\mu^{0}$ denotes the internal free energy of the corresponding cluster type.) The term $\ln \phi i$ comes from the entropy of mixing. The number $v_{A}^{G}$ of $A$-chains (per unit cell) participating in the gel network and the free-energy change $\delta_{A} \equiv \beta\left(\mu_{A}^{0}-\mu_{A}^{G 0}\right)$ which is produced when an isolated $A$-chain becomes a part of the network have been introduced. The parameter $\xi$ in front of the second term indicates the type of IPN; we have $\xi=1$ for a sim-IPN, while $\xi=0$ for a seq-IPN because an $A$-network is prepared in the reference state in a seq-IPN. A more de- tailed description of all these quantities is given in Ref. [8].

In order to find the distribution of clusters, we now apply our general theory [8] to the present binary mixtures. Let us first impose the multiple-equilibrium conditions for the association-dissociation process of clustering. These conditions can be written in terms of the chemical potentials of the clusters of various type derived from the free energy (1). Find the volume fraction of each cluster type as a function of $x \equiv \phi_{1}^{A}$ (or $y \equiv \phi_{1}^{B}$ ) of the corresponding isolated chains. By using the association constants $K_{l, 0}$ $=\exp \left(l-1-\Delta_{l}^{A}\right)$ and $K_{0, m}=\exp \left(m-1-\Delta_{m}^{B}\right)$, these relations are written as $\phi l^{A}=K_{l, 0} x^{l}$ and $\phi_{m}^{B}=K_{0, m} y^{m}$. They are not altered by the existence of the elastic terms in the free energy. The sol fraction of each species is then given by the infinite summation $\phi_{A}(x)=\sum_{l=1}^{\infty} K_{l, 0} x^{l}$ and $\phi_{B}(y)=\sum_{m=1}^{\infty} K_{0, m} y^{m}$.

The sol-to-gel transition point can then be found [8] from the convergence radius of the infinite series given above for each species. In the pregel regime of species $A$, for instance, we have $\phi_{A}(x)=\phi$, because there is no gel volume fraction. Solving this relation with respect to $x$, we can express $x$ in terms of the total composition; $x=x(\phi)$. Similarly in the pregel regime of species $B$, we have $y=y(\phi)$. Once $x$ or $y$ hits the convergence radius, however, it is fixed to a different function $x^{*}(\phi)$ $=\exp \left[\delta_{A}(\phi)-1\right]\left(\right.$ or $\left.y^{*}(\phi)=\exp \left[\delta_{B}(\phi)-1\right]\right)$ giving the radius of convergence of the infinite series. Suppose the composition $\phi$ is changed from 0 to 1 at a fixed temperature. In the small- $\phi$ region where $B$-chains are the majority, $A$-chains form a sol phase with finite clusters only, while there are a sufficiently large number of $B$-chains to form a macronetwork. In the large-composition region $\phi \simeq 1$, we have the opposite situation; an $A$-network is formed in the $B$-sol. Therefore the possibility arises that, in the intermediate composition region, an $A$-network and a $B$-network can simultaneously coexist at low temperatures. This is a bulk phase of sim-IPN. Because of the mutual immiscibility, however, a homogeneous IPN cannot remain a stable thermodynamic phase at most temperatures.

In order to study the phase stability, let us find the stability limit (spinodal line) which separates a thermodynamically unstable region from a stable one. This is found by the condition $\partial^{2} \Delta F / \partial \phi^{2}=0$, which takes the explicit form

$$
\gamma A^{\prime \prime}(\phi)+\xi \frac{\kappa_{A}(\phi)}{n_{A} \phi}+\frac{\kappa_{B}(\phi)}{n_{B}(1-\phi)}-2 \chi=0
$$

for the present system in the regime where there is neither $A$-gel nor $B$-gel. Here the $\kappa$ functions are given by the logarithmic derivative of $x$ and $y: \kappa_{A}(\phi) \equiv \phi \partial \ln x / \partial \phi$ and $\kappa_{B}(\phi) \equiv-(1-\phi) \partial \ln y / \partial \phi$. In the postgel regime, they must be replaced by corresponding postgel forms. The binodals (two-phase equilibria) are also found from the balancing conditions of the chemical potential of each 
species.

To find specific results, we have to specify the internal free energy $\Delta_{l}^{A}$ and $\Delta_{m}^{B}$ (or, equivalently, the association constants $K_{l, 0}$ and $K_{0, m}$ ) of the cluster formation. We introduce here the simplest model system in which each cluster is made in the form of a Cayley tree [12]. Internal loop formation is neglected. Simple combinatorial counting then gives $\lambda_{A} K_{l, 0}=\omega_{l}^{A}\left(f \lambda_{A}\right)^{\prime}$ and $\lambda_{B} K_{0, m}$ $=\omega_{m}^{B}\left(g \lambda_{B}\right)^{m}$ for the association constants, where $\lambda_{A}$ $\equiv \exp \left(1-\beta \Delta f_{A}\right)$ and $\lambda_{B} \equiv \exp \left(1-\beta \Delta f_{B}\right)$ depend only on the temperature. They are written in terms of the freeenergy change $\Delta f_{A}$ (and $\Delta f_{B}$ ) of $A$-bond (and $B$-bond) formation. The combinatorial coefficients are given by the Stockmayer distribution function [12] for each species: $\quad \omega_{l}^{A} \equiv(f l-l) ! f^{\prime} / l !(f l-2 l+2) !$ and $\omega_{m}^{B} \equiv(g m$ $-m) ! g^{m} / m !(g m-2 m+2) !$. We now use the scaled variables $x \equiv f \lambda_{A} \phi_{1}^{A}$ and $y \equiv g \lambda_{B} \phi_{1}^{B}$ instead of the original $x$ and $y$. Upon substitution of the combinatorial factor, the infinite series can be summed up to give the relations in the pregel regime:

$$
\lambda_{A} \phi=G_{0}^{f}(x), \lambda_{B}(1-\phi)=G_{0}^{g}(y),
$$

where $G f(x) \equiv \sum_{l=1}^{\infty} l^{k} \omega_{l}^{A} x^{l}(k=0,1,2,3, \ldots)$ is the $k$ th moment of the Stockmayer distribution. $\left[G_{k}^{g}(y)\right.$ is similarly defined.] Properties of these moments were explored in Ref. [12]. Specifically, the radius of convergence was found to be given by $x^{*} \equiv(f-2)^{f-2} /(f$ $-1)^{f-1}$ [or $y^{*} \equiv(g-2)^{g-2} /(g-1)^{g-1}$ ]. Since $\lambda$ 's are the functions of the temperature only, the relation $x=x^{*}$ (or $y=y^{*}$ ) decides the sol-to-gel transition line on the phase plane. Inside the radius of convergence, the function $G_{0}^{f}(x)$ [or $G_{0}^{g}(y)$ ] is a single-valued function and the relation (4) can be solved with respect to $x(y)$, the logarithmic derivative of which gives the function $\kappa_{A}\left(\kappa_{B}\right)$. As soon as $x(y)$ reaches $x^{*}\left(y^{*}\right)$ by increasing (decreas-

$\tau$

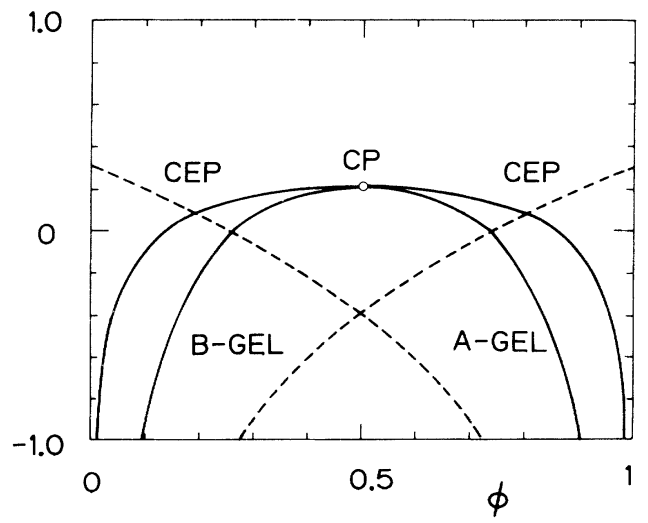

FIG. 1. Phase diagram of a symmetric low-molecular-weight sim-IPN. Binodal (outer solid line), spinodal (inner solid line), and gelation transitions (dashed lines) are shown. The critical point is indicated by an open circle. The entropy parameter $\lambda$ is changed from curve to curve. ing) the composition $\phi$, however, it remains constant at $x^{*}\left(y^{*}\right)$. The gel fraction is then given by $\phi_{A}^{G}$ $=\phi-n_{A}(f-1) /(f-2)^{2} \lambda_{A}$ lor $\phi_{B}^{G}=1-\phi-n_{B}(g-1) /$ $(g-2)^{2} \lambda_{B}$ ]. The $\kappa_{A}^{*}(\phi)$ [or $\kappa_{B}^{*}(\phi)$ ] therefore vanishes in its postgel regime.

In the following numerical calculation, the temperature is measured in terms of the reduced temperature deviation $\tau$ which is defined by $\tau \equiv 1-\theta / T$, where $\theta$ is the unperturbed theta temperature which satisfies the condition $\chi(\theta)=\frac{1}{2}$. Around this temperature, the interaction parameter $\chi$ is assumed to take the form $\chi(T)=\frac{1}{2}-\psi \tau$, where $\psi$ is a numerical parameter of order unity [7]. We then split the free energy $\Delta f$ of single-bond formation for each species into the energy and the entropy parts: $\Delta f=\Delta \epsilon-T \Delta s$. Hence the parameter $\lambda$ can be written in a form $\lambda=\lambda^{0} e^{r(1-r)}$, where the parameter $\lambda^{0} \equiv e^{1-\Delta s / k}$ includes the entropy change only, and $r \equiv-\Delta \epsilon / \Theta(>0)$ the binding energy measured relative to the theta temperature. In the following calculation we fix $\psi=1$ and $r_{A}=r_{B}=1$ for simplicity without losing any physical reality. We study how the phase behavior changes for different values of the entropy parameters $\lambda_{A}^{0}$ and $\lambda_{B}^{0}$.

Figure 1 shows a typical result for the low-molecularweight symmetric sim-IPN $(\xi=1) \quad\left(n_{A}=n_{B}=1\right)$ with functionality $f=g=3$. Solid lines show the binodals and the spinodals, while the dashed lines show the sol-to-gel transition lines. In the temperature region below the dashed lines, we have a gel of each species; $B$-gel in the low-concentration region and $A$-gel in the high-concentration region. In the overlapping region of the two, we have IPN. For a small $\lambda_{A}$ (such as $\lambda_{A}=1$ shown in this figure) the overlap region lies inside the spinodal, showing that the IPN cannot remain a stable phase. We have a critical solution point (CP) shown by an open circle in the middle of the spinodal line. The intersections between the spinodal and the gelation lines give critical end points, which were studied in detail in Ref. [13].

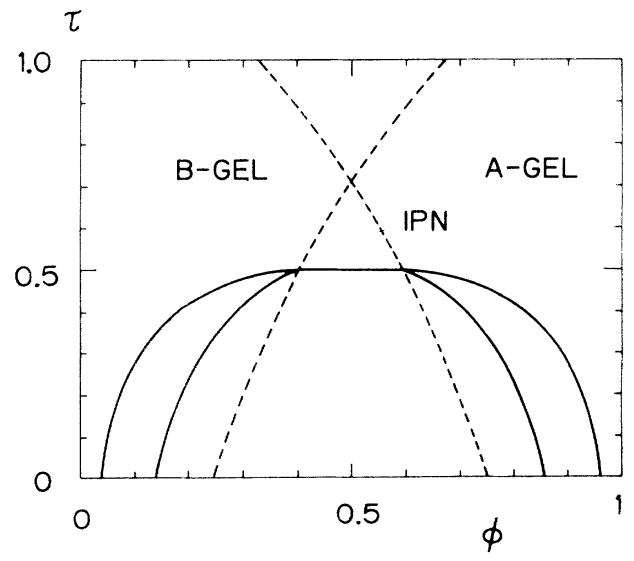

FIG. 2. Phase diagram of a symmetric sim-IPN for a larger value of $\lambda$. In the small triangular region above the top of the miscibility gap, the IPN is stabilized. 


\section{$\tau$}

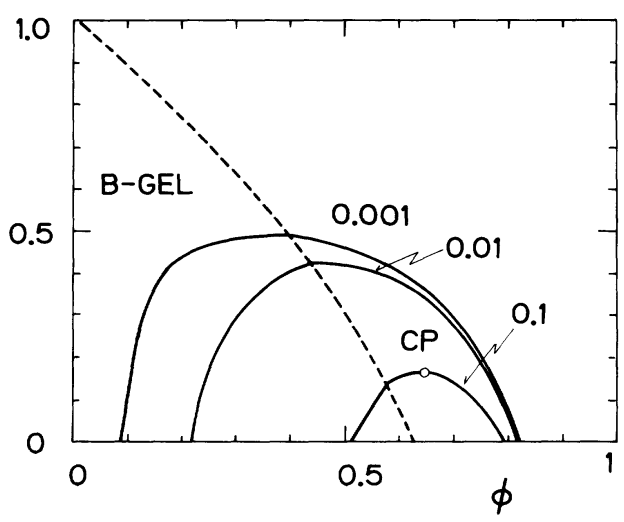

FIG. 3. Phase diagram of a low-molecular-weight seq-IPN. The number $\gamma$ of the elastically active chains in the $A$-network is changed from curve to curve. Stable IPN's are formed in the regions below the $B$-gel line (dashed line) and outside the spinodal (solid lines).

As the entropy parameter $\lambda$ is increased, the overlap region is enlarged and comes closer to the spinodal, and eventually the CP disappears; the top part of the overlap region moves out of the miscibility gap. Figure 2 shows the case $\lambda_{A}=3$ as an example. In this small triangular region, the IPN remains a stable homogeneous phase. The intersections between the gelation lines and the spinodal (and also the binodal) are the tricritical points (TCP). Because a larger entropy loss $\Delta s_{A}(<0)$ gives a larger value of $\lambda_{A}$, the existence of a stable IPN is more probable for the associative force which produces a large entropy loss when a bond is formed. Orientational restriction in the course of bond formation, such as seen in the hydrogen bonding for instance, can lead to a large entropy loss. The asymmetric high-molecular-weight simIPN, whose $n_{A}$ is much larger than $n_{B}$ for instance, can also be studied in a similar way. The miscibility gap shifts to lower concentration region as in the un-crosslinked polymer solutions, but the triangular IPN region remains essentially unchanged.

Figure 3 shows a typical result for a low-molecularweight seq-IPN $(\xi=0)$. Monomers $\left(n_{B}=1\right)$ carrying reactive groups (functionality $g=3$ ) are connected to each other to form a gel under the presence of a permanent $A$-network. The James-Guth theory $(B=0)$ has been employed for the elastic energy [14]. Solid lines show the spinodal boundaries, while the dashed line shows the sol-to-gel transition line of species $B$. The parameter $\lambda_{B}$ is fixed at 2 . Since we have a permanent $A$ network from the initial stage, the region below the dashed line and outside the spinodal gives a stable seqIPN. Spinodals for the three different values of $\gamma$-that is, the number of elastically active chains per unit cell - are shown in the figure. For calculational simplicity, and to remove the ambiguity, we have set $B=0$. The gelation line turns out to be independent of $\gamma$ in our molecular-field approximation. We have a single TCP only as shown in the figure. The high-molecular-weight seq-IPN can be discussed in a similar way.

In conclusion, the phase behavior of the reversible IPN's described in this study provides help in designing new IPN's and controlling their morphologies.

[1] L. H. Sperling, Interpenetrating Polymer Networks and Related Materials (Plenum, New York, 1981).

[2] L. H. Sperling, C. S. Heck, and J. H. An, in Multiphase Polymers: Blends and Ionomers, American Chemical Society Symposium Series Vol. 395 (American Chemical Society, Washington, DC, 1988), Chap. 9, p. 230.

[3] H. L. Frisch, British Polym. J. 17, 149 (1985).

[4] K. Binder and H. L. Frisch, J. Chem. Phys. 81, 2126 (1984).

[5] P. J. Flory, Principle of Polymer Chemistry (Cornell Univ. Press, Ithaca, NY, 1953), Chap. 11.

[6] F. Tanaka, M. Ishida, and A. Matsuyama, Macromolecules 24, 5582 (1991).

[7] Flory (Ref. [5]), Chap. 12.

[8] F. Tanaka, Macromolecules 23, 3784 (1990).

[9] H. M. James and E. Guth, J. Chem. Phys. 15, 669 (1947); 21, 1039 (1953).

[10] P. J. Flory, J. Chem. Phys. 18, 108 (1950).

[11] W. Kuhn and F. Grün, J. Polym. Sci. 1, 183 (1946).

[12] W. H. Stockmayer, J. Chem. Phys. 11, 45 (1943); 12 , 125 (1944).

[13] F. Tanaka, Macromolecules 22, 1988 (1989); F. Tanaka and A. Matsuyama, Phys. Rev. Lett. 62, 2759 (1989).

[14] Reference [4] showed that the phase stability depends strongly on whether $B$ vanishes or not. For $B=0$, all sim-IPN's were shown to be unstable and exhibit phase separation. In our calculation, however, there should be a certain temperature range in which a molecularly homogeneous phase is stabilized, provided that the strength of the cross-linking is judiciously chosen. 